\title{
Tanshinone IIA Induces Apoptosis in Fibroblast-Like Synoviocytes in Rheumatoid Arthritis via Blockade of the Cell Cycle in the G2/M Phase and a Mitochondrial Pathway
}

\author{
Ligang Jie, ${ }^{*, a, c, \#}$ Hongyan Du, ${ }^{b, \#}$ Qingchun Huang, ${ }^{d}$ Song Wei, ${ }^{a}$ Runyue Huang, ${ }^{d}$ and \\ Weifeng $\operatorname{Sun}^{*, a}$ \\ ${ }^{a}$ Department of Chinese Medicine, Guangzhou General Hospital of Guangzhou Command; PLA, Guangzhou \\ 510010, China: ${ }^{b}$ School of Biotechnology, Southern Medical University; Guangzhou 510515, China: ${ }^{c}$ HuaBo Bio- \\ Pharmaceutic Institute of Guangzhou; Guangzhou 510010, China: and ${ }^{d}$ Department of Rheumatology, The Second \\ Affiliated Hospital, Guangzhou University of Chinese Medicine (Guangdong Provincial Hospital of Chinese \\ Medicine); Guangzhou 510006, China.
}

Received April 12, 2014; accepted May 27, 2014; advance publication released online June 11, 2014

\begin{abstract}
Tanshinone IIA (Tan IIA), a phytochemical derived from the roots of Salvia miltiorrhiza Bunge, has been documented with anti-tumor, pro-apoptotic, and anti-inflammatory activities. Salvia miltiorrhiza has long been used to treat rheumatoid arthritis (RA). Apoptosis induction of RA-fibroblast-like synoviocytes (FLS) was suggested to be a potential therapeutic approach for RA. The aim of this study was to investigate whether Tan IIA promotes apoptosis in RA-affected FLS. In this study, the viability of an immortalized FLS cell line derived from RA patients was assessed by 3-(4,5-dimethylthiazol-2-yl)-5,3-carboxymethoxyphenyl-2,4sulfophenyl-2H-tetrazolium (MTS) assay after Tan IIA treatment. Apoptosis was measured by terminal deoxyuridine triphosphate (dUTP) nick-end labeling (TUNEL) assay and flow cytometry. Cell cycle was evaluated by flow cytometry. The expressions of mitochondrial apoptosis-related molecules, including Bcl-2, Bax, mitochondrial cytochrome $c$ (Cyt-c), cytosolic Cyt-c, apoptotic protease activating factor 1 (Apaf-1), procaspase-9, procaspase-3, caspase-9, and caspase-3 were determined by Western blotting. Our data demonstrate that Tan IIA induced apoptosis of RA-FLS, blocked the cell cycle in the G2/M phase, and regulated the protein expression of Bcl-2, Bax, and Apaf-1, the release of mitochondrial Cyt-c, and the activation of caspase-9 and caspase-3. The results support the conclusion Tan IIA treatment likely induces apoptosis of RA-FLS through blockade of the cell cycle in the G2/M phase and a mitochondrial pathway. These data suggest that Tan IIA may have therapeutic potential for RA.
\end{abstract}

Key words rheumatoid arthritis; fibroblast-like synoviocyte; Tanshinone IIA; apoptosis; cell cycle

Rheumatoid arthritis (RA) is a chronic inflammatory disease characterized by synovial hyperplasia and progressive joint destruction. ${ }^{1)}$ Although various cell populations may participate in the pathogenesis of RA, fibroblast-like synoviocytes (FLS) are considered crucial in both the initiation and progression of arthritis. The mechanisms of synovial hyperplasia are not fully understood, although the paucity of the apoptosis may contribute to the pathogenesis of RA. Recent evidence suggests that the activation of RA-FLS is associated with a reduced level of apoptosis in vivo, especially at sites of invasion into cartilage and bone. ${ }^{2)}$ Apoptosis induction of RAFLS is therefore suggested as a potential therapeutic approach for RA. ${ }^{3)}$

Salvia miltiorrhiza (Labiatae) is used primarily for the treatment of coronary heart diseases in China, and has long been used to treat arthritis. ${ }^{4,5}$ ) Tanshinone IIA (Tan IIA; 14,16-epoxy-20-nor-5(10),6,8,13,15-abietapentaene-11,12-dione) is a major component of Salvia miltiorrhiza and Chemical structure of Tan IIA is shown in Fig. 1. Moreover, recent studies have documented anti-tumor, pro-apoptotic, and anti-inflammatory activities of Tan IIA. ${ }^{6-8)}$ Tan IIA induces apoptosis in human cancer cell lines via the mitochondriadependent pathway involving Bcl-2, p53, and Bax. Activation of p53 leads to the release of many mitochondrial proteins, such as cytochrome $c$ (Cyt-c) via translocation of Bax from

The authors declare no conflict of interest.

\# These authors contributed equally to this work. the cytosol to mitochondria, overcoming the regulation by Bcl-2 of mitochondrial membrane protein permeability. An apoptosome complex then forms through the binding of Cyt-c, apoptotic protease activating factor 1 (Apaf-1), procaspase-9, and dATP. The apoptosome complex dimer then activates caspase-9 and further activates caspase-3, which degrade centrosome DNA by DNase activity to induce apoptosis. ${ }^{9-11)}$

Given the pathologic similarities between RA-FLS and tumor cells, ${ }^{2,12)}$ Tan IIA likely has similar effects on apoptosis in RA-FLS. However, despite the increasing evidence of the pro-apoptotic properties of Tan IIA, the effects of Tan IIA on

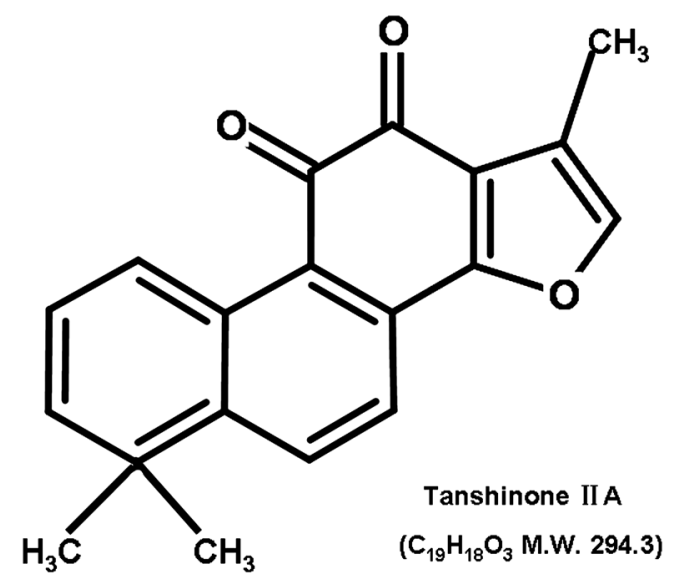

Fig. 1. Chemical Structure of Tanshinone IIA 
cellular signaling in RA have not yet been examined. We performed this study to investigate whether Tan IIA treatment of RA-FLS promotes apoptosis through blockade cell cycle and a mitochondrial pathway.

\section{MATERIALS AND METHODS}

Culture of RA-FLS RA-FLS were ordered from Cell Applications Inc. (San Diego, CA, U.S.A.). Cells were grown in Dulbecco's modified Eagle's medium (DMEM; Gibco BRL, Grand Island, U.S.A.) supplemented with fetal 10\% bovine serum (FBS; Gibco BRL), $100 \mathrm{U} / \mathrm{mL}$ penicillin, and $100 \mathrm{mg} / \mathrm{L}$ streptomycin. Cells were cultured at $37^{\circ} \mathrm{C}$ in a humidified incubator containing $5 \% \mathrm{CO}_{2}$. RA-FLS obtained from passages three to six were used for experiments.

Assessment of Cytotoxicity by 3-(4,5-Dimethylthiazol2-yl)-5,3-carboxymethoxyphenyl-2,4-sulfophenyl-2H-tetrazolium (MTS) Assay Tan IIA (purity $\geq 98 \%$; HPLC) was purchased from Shanghai Yuanye Biotech Co. (Shanghai, China) and dissolved in dimethyl sulfoxide (DMSO; Sigma). RA-FLS were seeded in 24-well plates at a density of $2 \times 10^{4}$ cells/well, and were treated with $2.5,5,10,20,40$, and $80 \mu \mathrm{M}$ Tan IIA or DMEM (control). After 24-, 48-, or 72-h incubation, $2.5 \mathrm{mg} / \mathrm{mL}$ MTS solution (Promega) was added to the wells, cells were incubated for $2 \mathrm{~h}$, and absorbance at $490 \mathrm{~nm}$ was measured using a microplate reader. MTS assay results informed the selection of Tan IIA concentrations that did not adversely affect cell viability for use in subsequent experiments.

Induction of RA-FLS Apoptosis by Terminal Deoxyuridine Triphosphate (dUTP) Nick-End Labeling (TUNEL) Assay After $48 \mathrm{~h}$ incubation in various Tan IIA concentrations, RA-FLS were detached and placed in serum-free medium for $24 \mathrm{~h}$. Apoptotic events were measured by an DeadEnd $^{\mathrm{TM}}$ Fluorometric TUNEL System (Promega) according to the manufacturer's protocol. Briefly, cells were seeded on glass cover slides previously coated with gelatin $1 \%$ in 24 wells plates and cultivated to reach $80 \%$ confluency. After proper treatment, culture medium was removed and fixation was performed with $4 \%$ neutral formalin in phosphate buffered saline (PBS) for $25 \mathrm{~min}$ at $4^{\circ} \mathrm{C}$. After washing two times with PBS, the cells were kept in $70 \%$ ethanol at $-20^{\circ} \mathrm{C}$ overnight. Cells were subsequently saturated and permeabilized with $0.2 \%$ Triton X-100 in PBS for $5 \mathrm{~min}$. Next, $100 \mu \mathrm{L}$ buffer including $45 \mu \mathrm{L}$ equilibration buffer, $5 \mu \mathrm{L}$ nucleotide Mix, and $1 \mu \mathrm{L}$ rTdT Enzyme was added for $10 \mathrm{~min}$. After washing 15 min with saline sodium citrate (SSC) and two times with PBS, cells were incubated for 4',6-diamidino-2-phenylindole (DAPI) staining at room temperature for $15 \mathrm{~min}$ at dark. Acquisition of the images was performed with a fluorescence microscope. Cells in each slide were counted in no less than five fields, and the apoptosis ratio was calculated as the number of positive cells divided by the number of DAPI-staining cells.

Induction of RA-FLS Apoptosis by Flow Cytometry After $48 \mathrm{~h}$ incubation in various Tan IIA concentrations, RAFLS were detached and placed in serum-free medium for $24 \mathrm{~h}$. Apoptosis was analyzed by two-dimensional flow cytometry using annexin $\mathrm{V}$ and propidium iodide (PI), according to the manufacturer's instructions (Keygen), in a Becton Dickinson FACSort flow cytometer (Becton Dickinson, San Jose, CA, U.S.A.). Dead cells were not gated out; rather, after gating out debris, RA-FLS were gated for an annexin V-positive versus PI-positive contour plot. Numbers in the dot plots represent the percentages of annexin V-positive, PI-positive cells and annexin V-positive, PI-negative cells. Data are expressed as mean \pm S.D. of three independent experiments.

Analysis of Cell Cycle by Flow Cytometry After $48 \mathrm{~h}$ incubation in various Tan IIA concentrations, RA-FLS of all groups were digested and collected using $0.25 \%$ trypsin, and then washed with PBS solution. Cells were fixed at $4{ }^{\circ} \mathrm{C}$ with $75 \%$ cold ethanol overnight and washed with PBS solution. The cell density was adjusted to $1 \times 10^{6}$ cells $/ \mathrm{mL}$ and the final volume was $100 \mu \mathrm{L}$. DNAStain comprehensive dye liquor (500 mL; Sigma, St. Louis, MO, U.S.A.) was added for storage at room temperature in a dark place for $30 \mathrm{~min}$ prior to testing with flow cytometry. The DNAStain contained RNase, PI and Triton X-100 at end concentrations of $50 \mathrm{mg} / \mathrm{L}, 100 \mathrm{mg} / \mathrm{L}$, and $1 \mathrm{~mL} / \mathrm{L}$, respectively.

Western Blot Analysis After $48 \mathrm{~h}$ incubation in various Tan IIA concentrations, RA-FLS were washed with ice-cold PBS, collected and homogenized with radio immunoprecipitation assay (RIPA) lysis buffer containing $1 \times$ PBS, $1 \%$ Nonidet P-40, $0.5 \%$ sodium deoxycholate, $0.1 \%$ sodium dodecyl sulfate (SDS), and proteinase inhibitors. Total protein was extracted and measured by the Bio-Rad protein assay (Bio-Rad Laboratories, Hercules, CA, U.S.A.). Equal amounts of proteins $(50 \mu \mathrm{g})$ were subjected to $10 \%$ SDS-polyacrylamide gel electrophoresis (PAGE) and then transferred onto polyvinylidene difluoride (PVDF) membranes (Millipore, Billerica, MA, U.S.A.). The following antibodies were used for the Western blot analysis: a mouse monoclonal antibody $(1: 600)$ against Bcl-2, Bax, Apaf-1, caspase-3, caspase-9, procaspase-3, and procaspase-9 obtained from Santa Cruz Biotechnology (Santa Cruz, CA, U.S.A.), and a rabbit polyclonal antibody $(1: 1000)$ against $\beta$-actin was bought from Cell Signaling Technology (Beverly, MA, U.S.A.). Immunoreactive proteins were detected using the ELC Western blot detection system kit (Amersham, Braunschweig, Germany). Band intensity was analyzed

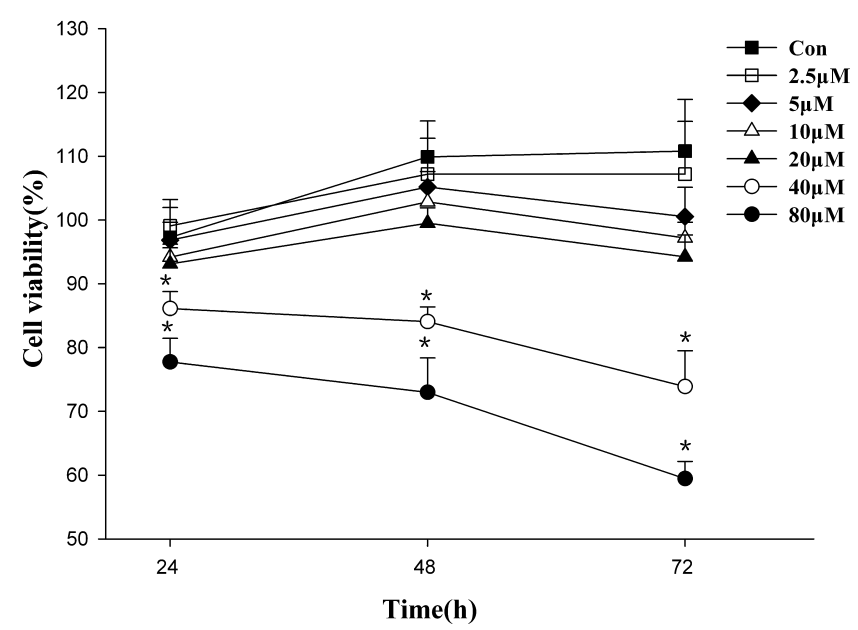

Fig. 2. Cell Viability Rate of Tan IIA on RA-FLS Detected by a MTS Assay

RA-FLS were treated with different concentrations of Tan IIA $(2.5,5,10,20,40$, and $80 \mu \mathrm{M}$ ), and Con (control group cells were treated with DMEM using the same volume as Tan IIA treatment). The cell viability rate were analyzed at three time points $(24,48$, and $72 \mathrm{~h})$ using a MTS assay. No changes of RA-FLS viability at concentrations from 2.5 to $20 \mu \mathrm{m}$ of Tan IIA were observed, compared to vehicletreated control cells $(p>0.05)$. Significant decreased cell viability were noted at both 40 and $80 \mu \mathrm{M}$ of Tan IIA for 24,48 , and $72 \mathrm{~h}, * p<0.05$ versus control. 
A

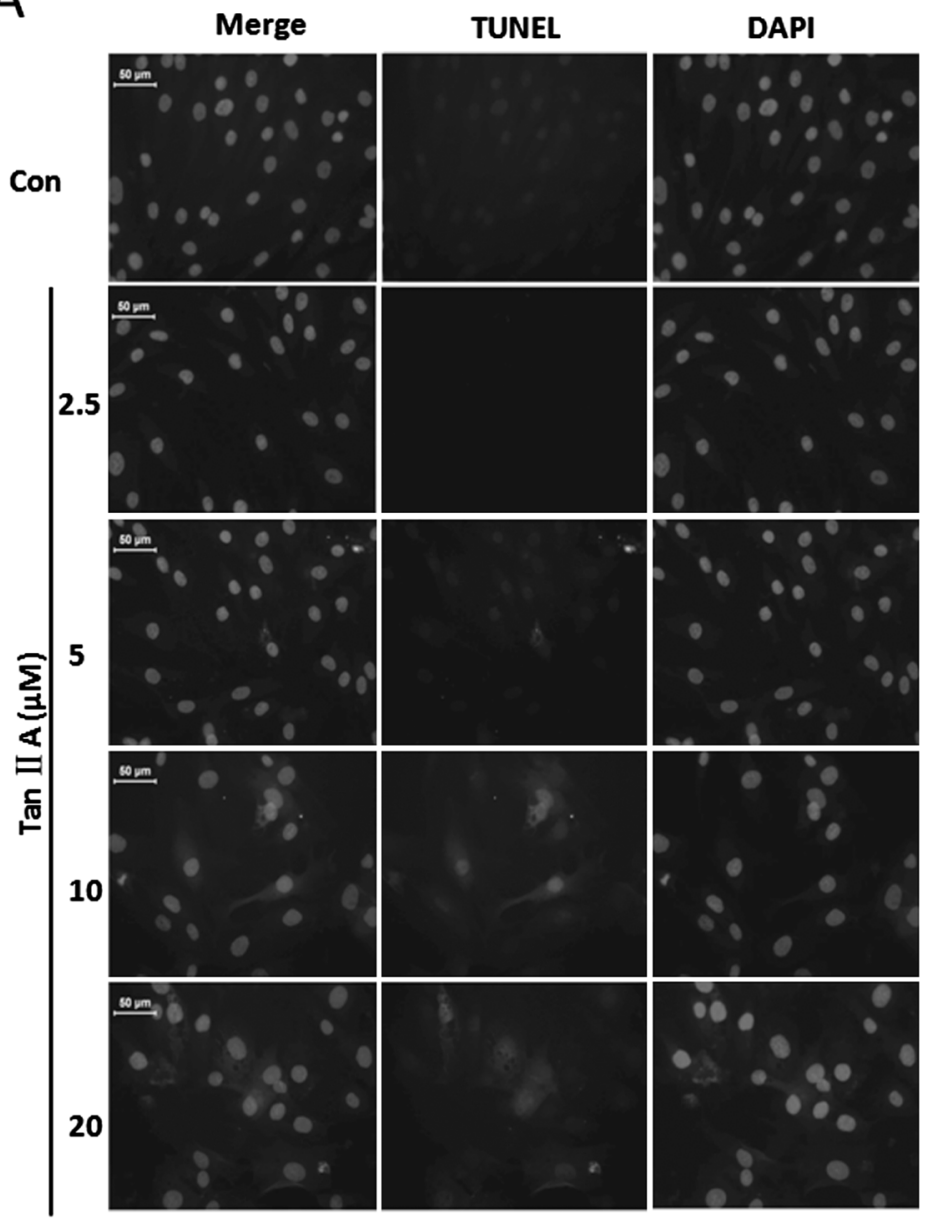

B

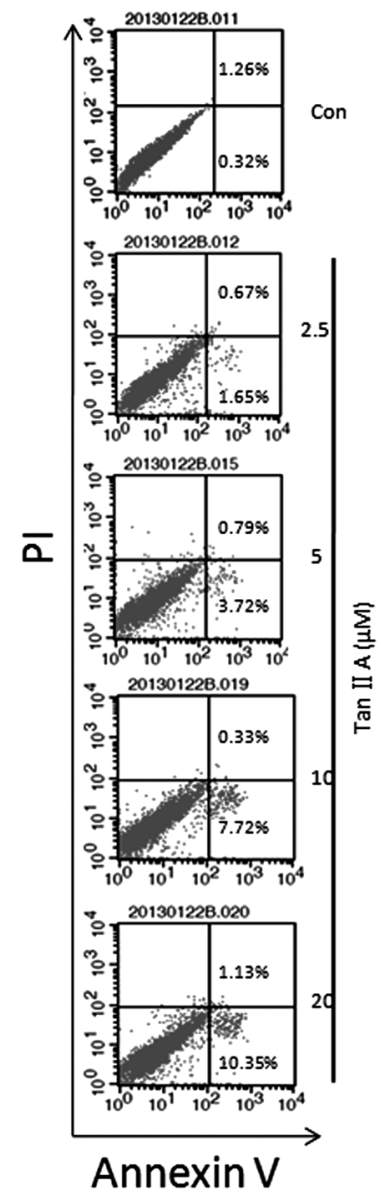

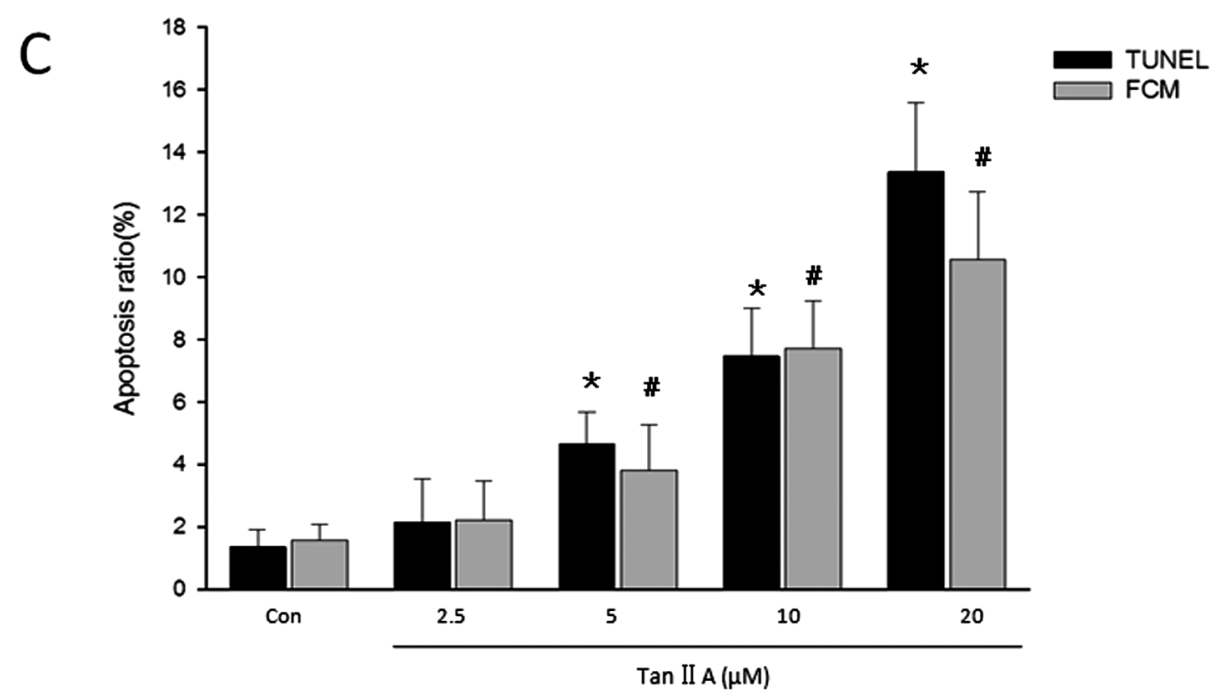

Fig. 3. Pro-apoptotic Effect of Tan IIA on RA-FLS in the Analysis of TUNEL Stain and Flow Cytometry Assay

(A) Fluorometric image of apoptotic cells. Blue, DAPI staining; Green, apoptotic positive staining. (Color images were converted into gray scale.) Tan IIA ( 5 , 10 and $20 \mu \mathrm{M})$ treatment enhanced the rate of apoptotic cells, $* p<0.05$ versus control treated with vehicle, shown in (C). (B) Apoptotic rate was evaluated by flow cytometry. Events accumulating in the top and bottom right-hand quadrants were counted as apoptotic cells. Apoptotic percentage of RA-FLS increased in accord with concentration of Tan IIA, ${ }^{\#} p<0.05$ versus control treated with vehicle as shown in (C).

and quantified using the Quantity One software (Bio-Rad, Hercules, CA, U.S.A.).

Cyt-c Release Measurements by Western Blot Analysis RA-FLS treatment with various concentrations of Tan IIA ( 0 , $2.5,5,10$, or $20 \mu \mathrm{M})$ for $48 \mathrm{~h}$. Release of Cyt-c from mitochon- dria to cytosol was measured by Western blot analysis. Cells were harvested, washed once with ice-cold phosphate-buffered saline and gently lysed for $30 \mathrm{~s}$ in $80 \mathrm{~mL}$ ice-cold lysis buffer $(250 \mathrm{~mm}$ sucrose, $1 \mathrm{~mm}$ ethylenediaminetetraacetic acid (EDTA), 0.05\% digitonin, $25 \mathrm{~mm}$ Tris, $\mathrm{pH} 6.8,1 \mathrm{~mm}$ dithio- 
A
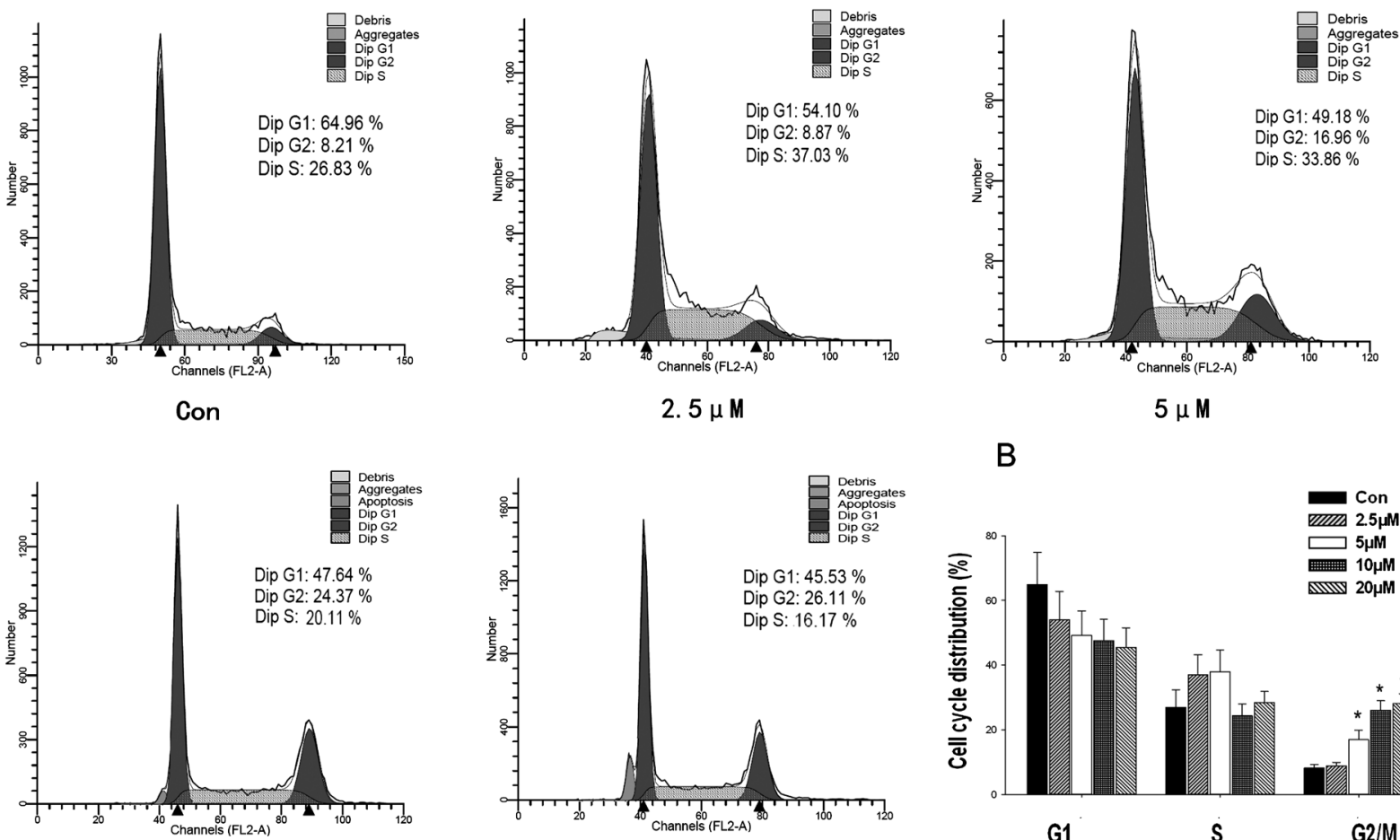

B

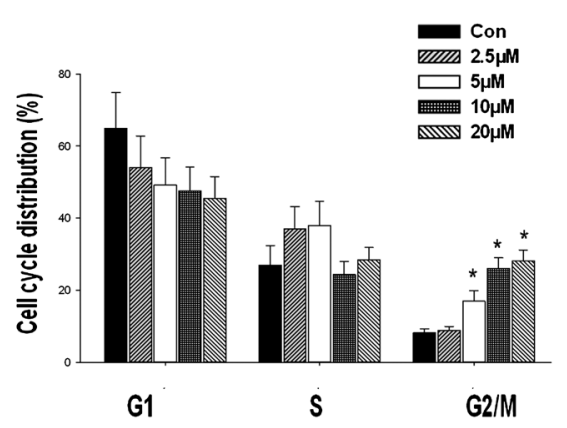

Fig. 4. Effect of Tan IIA on the Cell Cycle of RA-FLS

RA-FLS were treated with Tan IIA $(2.5,5,10$, and $20 \mu \mathrm{m}$ ) for $48 \mathrm{~h}$. (A) Flow cytometry was used to analyze the cell cycle. (B) The histogram shows the percentage of cells in $\mathrm{G} 2 / \mathrm{M}$ phase of the cell cycle. Data are expressed as mean \pm S.D. of three independent experiments done in triplicate. $* p<0.05 v s$. control.

threitol, $1 \mathrm{mg} / \mathrm{mL}$ leupeptin, $1 \mathrm{mg} / \mathrm{mL}$ pepstatin, $1 \mathrm{mg} / \mathrm{mL}$ aprotinin, $1 \mathrm{~mm}$ benzamidine, and $0.1 \mathrm{~mm}$ phenylmethylsulfonyl fluoride). Lysates were centrifuged at $12000 \times \boldsymbol{g}$ at $4 \mathrm{a}$ for $3 \mathrm{~min}$ to obtain the supernatant (cytosolic extracts free of mitochondria) and the pellets (fraction that contains mitochondria). Supernatants $(40 \mathrm{mg})$ and pellets $(40 \mathrm{mg})$ were electrophoresed on 15\% SDS-PAGE and then analyzed by Western blot using anti-cytochrome $c$ antibody (Santa Cruz, CA, U.S.A.).

Statistical Analysis All statistical tests were performed using the SPSS statistical software version 13.0 for Windows (SPSS Inc., Chicago, IL, U.S.A.). The level of statistical significance was determined by one-way ANOVA, followed by Bonferroni correction for multiple comparisons. The results are presented as means \pm standard error of the mean (S.E.M.) and a $p$ value of less than 0.05 with a $95 \%$ CI was considered to indicate significance.

\section{RESULTS}

Effects of Tan IIA on Cytotoxicity of RA-FLS To investigate the effect of Tan IIA treatment on cytotoxicity, RA-FLS were incubated with various concentrations of Tan IIA $(0,2.5,5,10,20,40$, or $80 \mu \mathrm{M})$ for 24,48 , or $72 \mathrm{~h}$ (Fig. 2). No significant suppression of cell viability was observed at up to $20 \mu \mathrm{M}$ Tan IIA, compared with vehicle-treated control cells $(p>0.05)$. However, Tan IIA treatment at 40 and $80 \mu \mathrm{M}$ significantly suppressed RA-FLS viability in a dose-dependent manner $(p<0.05)$; treatment with 40 and $80 \mu \mathrm{M}$ Tan IIA decreased cell survival by 88 and $75 \%$ after $24 \mathrm{~h}, 86$ and $72 \%$ of after $48 \mathrm{~h}$, and 76 and and $58 \%$ of after $48 \mathrm{~h}$, respectively.
As they did not adversely affect cell viability, we employed Tan IIA concentrations of $2.5,5,10$, and $20 \mu \mathrm{M}$ for subsequent experiments.

Induction of RA-FLS Apoptosis by Tan IIA We evaluated whether Tan IIA treatment influences the rate of apoptotic death of RA-FLS. Tan IIA treatment at 5, 10 and $20 \mu \mathrm{M}$ for $48 \mathrm{~h}$ increased the number of TUNEL-positive cells, compared to vehicle-treated control cells $(p<0.05)$ (Fig. 3A). The apoptosis rates of cells treated with 5,10 , and $20 \mu \mathrm{M}$ Tan IIA were $4.67 \pm 1.02 \%, 7.46 \pm 1.55 \%$, and $13.38 \pm 2.21 \%$, respectively. We also determined the pro-apoptotic effects of Tan IIA following treatment of RA-FLS using a flow cytometry assay (Fig. 3B). Tan IIA (5, 10 and $20 \mu \mathrm{M})$ treatment enhanced the rate of apoptotic cells, compared to vehicle-treated control cells $(p<0.05)$ (Fig. 3C). These data suggest a pro-apoptotic effect of Tan IIA on RA-FLS.

Induction of RA-FLS Cell Cycle Blockade in G2/M Phase by Tan IIA Flow cytometry was conducted to investigate whether Tan IIA affected the cell cycle of RA-FLS. The results revealed that $48 \mathrm{~h}$ following the addition of different concentrations $(5,10,20 \mu \mathrm{M})$ of Tan IIA to RA-FLS, a cell cycle blockade was observed in the $\mathrm{G} 2 / \mathrm{M}$ phase compared with the control group (Fig. 4). This suggests that Tan IIA is able to increase the percentage of RA-FLS in the G2/M phase to induce apoptosis of RA-FLS.

Regulation of Mitochondrial Apoptosis Pathway-Related Proteins by Tan IIA We next investigated the effect of Tan IIA treatment on the levels of several mitochondrial apoptosis pathway-related proteins, which are important factors for RAFLS resistance to apoptotic cell death. RA-FLS were treated with 0 to $20 \mu \mathrm{M}$ Tan IIA for $48 \mathrm{~h}$ and the levels of Bcl-2, Bax, 
A

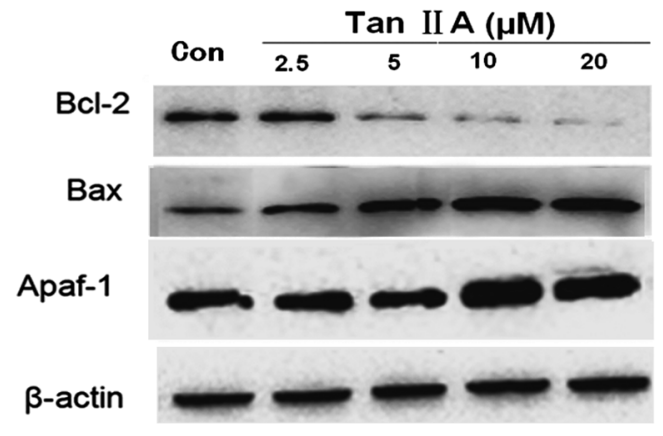

B
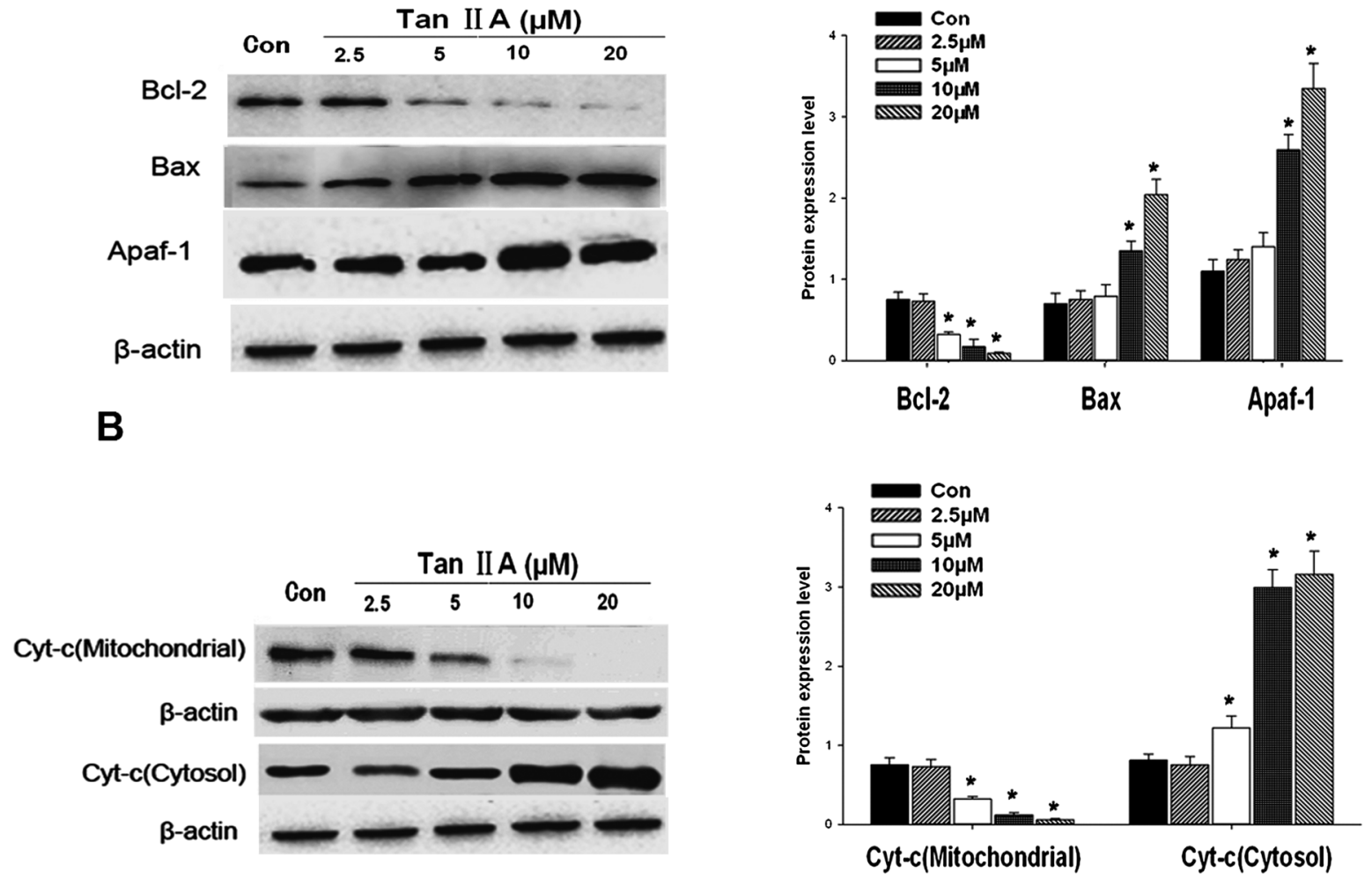

C
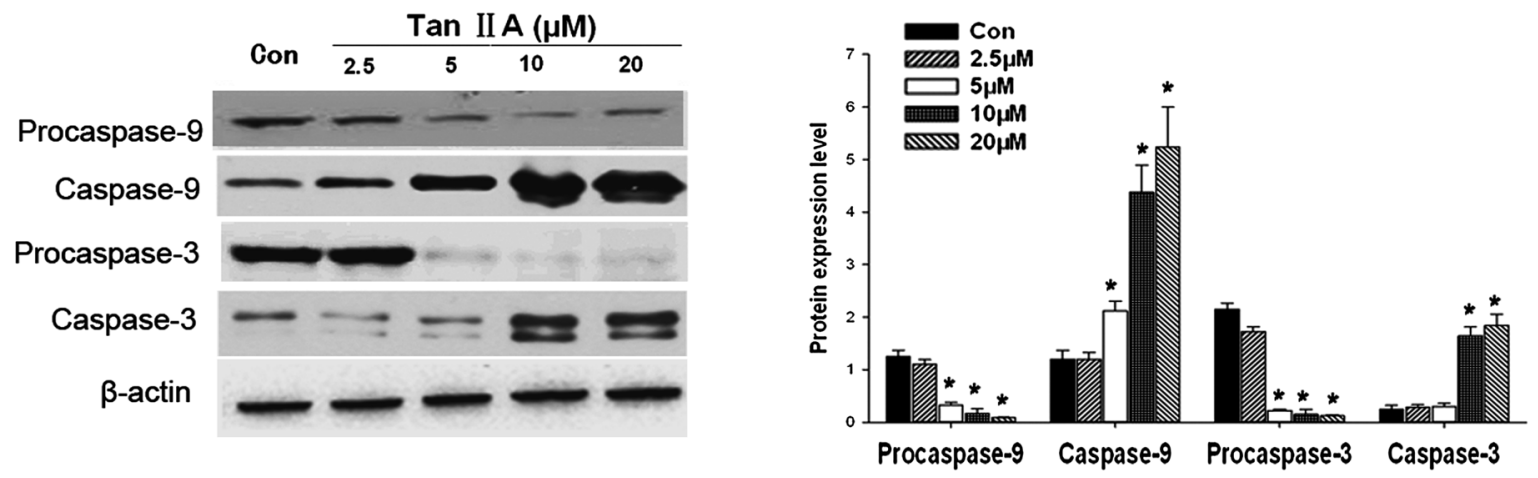

Fig. 5. Regulation of Mitochondrial Apoptosis Pathway-Related Proteins by Tan IIA in RA-FLS

RA-FLS were treated with Tan A $(2.5,5,10$ and $20 \mu \mathrm{M})$ for $48 \mathrm{~h}$. (A) Western blot analysis was performed to detect the protein levels of Bcl-2, Bax, Apaf-1. (B) The expression levels of mitochondrial cytochrome $c$ and cytosol cytochrome $c$ were detected by Western blot analysis, and $\beta$-actin was used as a loading control. (C) Western blot analysis was used to analyze the expression of procaspase-3, -9 and caspase-3, -9 proteins. (D) Graph represents means \pm S.D. of percent of protein levels of Bcl-2, Bax, Apaf-1, ${ }^{*} p<0.05$ vs. control. (E) The histogram shows the expression levels of mitochondrial cytochrome $c$ and cytosol cytochrome $c$. Data are means \pm S.D., $* p<0.05 v s$. control. (F) The graph represents means \pm S.D. of percent of protein levels of procaspase-3, -9 and caspase-3, $-9, * p<0.05 v s$. control. Data are determined in three independent experiments.

Apaf-1, cytosolic Cyt-c, mitochondrial Cyt-c, procaspase-9, procaspase-3, caspase-9, and caspase-3 were evaluated by Western blotting. As shown in Fig. 5A, the level of antiapoptotic Bcl- 2 were reduced by Tan IIA $(5,10$, and $20 \mu \mathrm{M})$, compared with vehicle-treated control cells $(p<0.05)$. The level of the proapoptotic Bax, Apaf-1 proteins were increased by Tan IIA (10 and $20 \mu \mathrm{M}$ ), compared with vehicle-treated control cells $(p<0.05)$; both responses were dose-dependent. As shown in Fig. 5B, Tan IIA induced Cyt-c release in a dosedependent manner. Both a significant increase in the amount of Cyt-c in the cytosol and a significant decrease in the mitochondria were detected. As shown in Fig. 5C, expression level of caspase- 9 proteins was significantly increased by Tan IIA at concentrations of 5, 10, and $20 \mu \mathrm{M}(p<0.05)$, and those of caspase- 3 was increased by Tan IIA at concentrations of 10 and $20 \mu \mathrm{M}(p<0.05)$, compared with vehicle-treated control cells $(p<0.05)$. Expression level of procaspase-9, -3 proteins was significantly decreased by Tan IIA at concentrations of 5,10 , and $20 \mu \mathrm{M}(p<0.05)$. These results indicate that Tan IIA treatment induces apoptosis of RA-FLS by regulating the production of pro/anti-apoptotic mitochondrial pathway-related proteins; these effects were also dose-dependent.

\section{DISCUSSION}

RA is a chronic inflammatory joint disease characterized 
by synovial proliferation and erosion of the affected joint. During the past few decades, drugs therapy for RA were mainly based on two principal approaches: symptomatic treatment with non-steroidal anti-inflammatory drugs and diseasemodifying anti-rheumatic drugs. ${ }^{13)}$ However, most of the currently available drugs were primarily directed towards the control of pain and/or the inflammation associated with joint synovitis, while did little to interfere with the underlying immunoinflammatory events, and consequently also did little to block the disease progression and reduce cartilage and bone destruction of joint, meanwhile, long-term side-effects and toxicity, high cost and incur hypersensitivity to medications and infections were also existence. ${ }^{14)}$ Therefore, there was a dramatically growing interest in Chinese traditional medicines in therapy for RA. In fact, Chinese traditional medicines were widely used virtually around the world for treatment inflammatory diseases and in recent few decades considerable advance have been made in both clinical and basic research on the treatment of autoimmune disease. ${ }^{15)}$ Salvia miltiorrhiza has been used widely in China for the treatment of RA. ${ }^{16)}$ Danshen (Salvia miltiorrhiza) injection inhibits proliferation of FLS in RA. ${ }^{17)}$ Tan IIA is a major component of $S$. miltiorrhiza and exhibits antitumor activities in many human cancer cell lines. Numerous studies demonstrate that Tan IIA also has pro-apoptotic activity, ${ }^{18,19)}$ and the present work was undertaken to elucidate the effects of Tan IIA on apoptotic death of FLS derived from RA-affected joints.

Synovial hyperplasia is one of the major pathological characteristics of RA, and leads to marginal bony erosions and resultant joint destruction. Several lines of evidence suggest that RA-FLS are resistant to apoptotic signaling, yielding expansion capabilities similar to tumors. Therefore, previous studies suggested that induction of apoptosis was a promising therapeutic strategy to eliminate RA synovial pannus. ${ }^{20)}$ In the present study, the result indicated that Tan IIA treatment could effectively induce apoptosis in RA-FLS in a dose-dependent manner. Moreover, Tan IIA has the potent effect of restraining the cell cycle transition of RA-FLS to blockade the cell cycle in $\mathrm{G} 2 / \mathrm{M}$ phase, therefore altering the progression of the cell cycle to induce apoptosis.

Apoptosis, or programmed cell death, results from various signaling pathways. The mitochondrial pathway is one of the most important signaling pathways. Bcl-2 family proteins are key regulatory factors of the mitochondrial pathway. ${ }^{21,22)} \mathrm{We}$ demonstrated that that when RA-FLS had been treated with different doses of Tan IIA for 48h, Bax and Bcl-2 migrated, the mitochondrial membrane potential increase expression of Bax, while Bcl-2 expression decreased and Cyt-c was released. These results indicated that the induction of RAFLS apoptosis by Tan IIA was closely associated with the mitochondrial pathway. As demonstrated in previous studies, with the stimulation of pro-apoptosis factors, the Bax protein migrated from the cytoplasm to the outer mitochondrial membrane, changing the permeability of the outer mitochondrial membrane to promote the mitochondrial release of Cyt-c. ${ }^{23)}$ Release of Cyt-c leads to the formation of the apoptosome complex by the binding of Cyt-c to Apaf-1, procaspase- 9 and dATP. The dimer complex activates first caspase- 9 and then caspase- $3,{ }^{24)}$ and these activites results in apoptosis. In the present study, We analyzed the changes in Bcl-2, Bax, Apaf-1, cytosolic Cyt-c, mitochondrial Cyt-c, procaspase-9, procas- pase-3, caspase-9, and caspase-3 proteins following treatment of RA-FLS with Tan IIA. A significant decrease in Bcl-2, mitochondrial Cyt-c, procaspase-3, and -9 expression and a significant increase in Bax, Apaf-1, cytosolic Cyt-c, caspase-3, and -9 expression were observed when RA-FLS apoptosis occurred. These results suggest that the pro-apoptotic effect of Tan IIA on RA-FLS is realized via the endogenous mitochondrial pathway

In conclusion, our results demonstrate that Tan IIA stimulates apoptosis of RA-FLS through a mitochondrial pathway in vitro. These findings may lead to the development of Tan IIA as a novel therapeutic agent for RA. We are aware of the limitations of this study, which assessed apoptosis without simulating a pathological condition. Further research to establish the in vivo relevance of these findings is warranted. Moreover, Tan IIA has a variety of pharmacological activities and biological targets; therefore, further study to identify the targets responsible for its pro-apoptotic effects is necessary.

Acknowledgments This study was supported by National Natural Science Foundation of China (No. 81102688) and Postdoctoral Science Foundation of China (No. 2012M521584). We thank Guangzhou Land Biotech Co., Ltd. for the technique assistance.

\section{REFERENCES}

1) McInnes IB, Schett G. The pathogenesis of rheumatoid arthritis. Engl. J. Med., 365, 2205-2219 (2011).

2) Bartok B, Firestein GS. Fibroblast-like synoviocytes: key effector cells in rheumatoid arthritis. Immunol. Rev., 233, 233-255 (2010).

3) Noss EH, Brenner MB. The role and therapeutic implications of fibroblast-like synoviocytes in inflammation and cartilage erosion in rheumatoid arthritis. Immunol. Rev., 223, 252-270 (2008).

4) Zhou L, Zuo Z, Chow MS. Danshen: an overview of its chemistry, pharmacology, pharmacokinetics, and clinical use. J. Clin. Pharmacol., 45, 1345-1359 (2005).

5) Cheng TO. Danshen: a popular chinese cardiac herbal drug. J. Am. Coll. Cardiol., 47, 1498, author reply 1499-1500 (2006).

6) Yin X, Yin Y, Cao FL, Chen YF, Peng Y, Hou WG, Sun SK, Luo ZJ. Tanshinone IIA attenuates the inflammatory response and apoptosis after traumatic injury of the spinal cord in adult rats. PLoS ONE, 7, e38381 (2012).

7) Yun SM, Jeong SJ, Kim JH, Jung JH, Lee HJ, Sohn EJ, Lee MH, Kim SH. Activation of c-Jun N-terminal kinase mediates tanshinone IIA-induced apoptosis in KBM-5 chronic myeloid leukemia cells. Biol. Pharm. Bull., 36, 208-214 (2013).

8) Won SH, Lee HJ, Jeong SJ, Lee HJ, Lee EO, Jung DB, Shin JM, Kwon TR, Yun SM, Lee MH, Choi SH, Lü J, Kim SH. Tanshinone IIA induces mitochondria dependent apoptosis in prostate cancer cells in association with an inhibition of phosphoinositide 3-kinase/ AKT pathway. Biol. Pharm. Bull., 33, 1828-1834 (2010).

9) Cheng CY, Su CC. Tanshinone IIA inhibits Hep-J5 cells by increasing calreticulin, caspase 12 and GADD153 protein expression. Mol. Med. Rep., 3, 645-650 (2010).

10) Li Y, Bian L, Cui FD, Li LC, Zhang XW. TTF1-induced apoptosis of HepG-2 cells through a mitochondrial pathway. Oncol. Rep., 26, 651-657 (2011).

11) Li JH, Xu M, Xie XY, Fan QX, Mu DG, Zhang Y, Cao FL, Wang YX, Zhao PT, Zhang B, Jin FG, Li ZC. Tanshinone IIA suppresses lung injury and apoptosis, and modulates protein kinase $\mathrm{B}$ and extracellular signal-regulated protein kinase pathways in rats challenged with seawater exposure. Clin. Exp. Pharmacol. Physiol., 38, 269-277 (2011). 
12) Liu FL, Wu CC, Chang DM. TACE-dependent amphiregulin release is induced by IL- $1 \beta$ and promotes cell invasion in fibroblast-like synoviocytes in rheumatoid arthritis. Rheumatology (Oxford), 53, 260-269 (2014).

13) Majithia V, Geraci SA. Rheumatoid arthritis: diagnosis and management. Am. J. Med., 120, 936-939 (2007)

14) Feldmann M, Brennan F, Bondeson J, Paleolog E, Foxwell B, Maini R. Analysis of cytokine expression in rheumatoid synovium has provided new insights into the pathogenesis of rheumatoid arthritis and new therapeutic opportunities. Transplant. Proc., 33, 20852086 (2001).

15) Liu M, Mao W, Guan H, Li L, Wei B, Li P. Effects of taurochenodeoxycholic acid on adjuvant arthritis in rats. Int. Immunopharmacol., 11, 2150-2158 (2011)

16) Huang QC, Chu YL, He XH, Huang RY. Regulatory roles of compound danshen in the downstream path of cyclooxygenases in rheumatoid arthritis patients' synovium. Zhongguo Zhong Xi Yi Jie He Za Zhi, 33, 1416-1419 (2013).

17) Liu QS, Zhu XC, Li JA, Xing Y, Jiang H, Zhang J, Yang MH, Zhao MC, Yuan GH, Tang Z. Effects of danshen injection on the proliferation of rheumatoid arthritis fibroblast-like synoviocytes cultured with human serum. Zhongguo Zhong Xi Yi Jie He Za Zhi, 33, 674-678 (2013).

18) Wang JG, Bondy SC, Zhou L, Yang FZ, Ding ZG, Hu Y, Tian
Y, Wen PY, Luo H, Wang F, Li WW, Zhou J. Protective effect of Tanshinone IIA against infarct size and increased HMGB1, NF $\kappa$ B, GFAP and apoptosis consequent to transient middle cerebral artery occlusion. Neurochem. Res., 39, 295-304 (2014).

19) Yun SM, Jeong SJ, Kim JH, Jung JH, Lee HJ, Sohn EJ, Lee MH, Kim SH. Activation of c-Jun N-terminal kinase mediates tanshinone IIA-induced apoptosis in KBM-5 chronic myeloid leukemia cells. Biol. Pharm. Bull., 36, 208-214 (2013).

20) Kim WU, Kwok SK, Hong KH, Yoo SA, Kong JS, Choe J, Cho CS. Soluble Fas ligand inhibits angiogenesis in rheumatoid arthritis. Arthritis Res. Ther., 9, R42 (2007).

21) Tang B, Zhang Y, Liang R, Yuan P, Du J, Wang H, Wang L. Activation of the $\delta$-opioid receptor inhibits serum deprivation-induced apoptosis of human liver cells via the activation of PKC and the mitochondrial pathway. Int. J. Mol. Med., 28, 1077-1085 (2011).

22) Mattson MP, Kroemer G. Mitochondria in cell death: novel targets for neuroprotection and cardioprotection. Trends Mol. Med., 9, 196-205 (2003).

23) Burlacu A. Regulation of apoptosis by Bcl-2 family proteins. $J$. Cell. Mol. Med., 7, 249-257 (2003).

24) Saito M, Korsmeyer SJ, Schlesinger PH. BAX-dependent transport of cytochrome $c$ reconstituted in pure liposomes. Nat. Cell Biol., 2 , 553-555 (2000). 